\title{
Data integrity in regulated bioanalysis: a summary from the European Bioanalysis Forum Workshop in collaboration with the MHRA
}

\author{
Cecilia Arfvidsson ${ }^{1}$, David Van Bedaf ${ }^{2}$, Mira Doig ${ }^{3}$, Susanne Globig ${ }^{4}$, Magnus Knutsson ${ }^{5}$, \\ Mark Lewis ${ }^{6}$, Stuart McDougall ${ }^{7}$, Marco Michi ${ }^{8}$, Nathalie Mokrzycki ${ }^{9} \&$ Philip \\ Timmerman*,10 \\ ${ }^{1}$ AstraZeneca R\&D, Gothenburg, Sweden \\ ${ }^{2}$ Janssen R\&D, Beerse, Belgium \\ ${ }^{3}$ ABS Laboratories, Welwyn Garden City, UK \\ ${ }^{4}$ Idorsia Pharmaceuticals Ltd, Allschwil, Switzerland \\ ${ }^{5}$ Ferring, Copenhagen, Denmark \\ ${ }^{6}$ GlaxoSmithKline R\&D, Ware, UK \\ ${ }^{7}$ ARCinova, Alnwick, UK \\ ${ }^{8}$ Aptuit an Evotec Company, Verona, Italy \\ ${ }^{9} \mathrm{MSD}$, Clermont-Ferrand, France \\ ${ }^{10}$ European Bioanalysis Forum vzW (EBF), Havenlaan 86c b204, 1000 Brussel, Belgium \\ *Author for correspondence: chair@e-b-f.eu
}

In this conference report, we summarize the main findings and messages from a workshop on 'Data Integrity'. The workshop was held at the 11th European Bioanalysis Forum Open (EBF) Symposium in Barcelona (21-23 November 2018), in collaboration with the Medicines and Health products Regulatory Agency to provide insight and understanding of regulatory data integrity expectations. The workshop highlighted the importance of engaging with software developers to address the gap between industry's data integrity needs and current system software capabilities. Delegates were also made aware of the importance of implementing additional procedural controls to mitigate the risk associated with using systems that do not fully meet data integrity requirements.

First draft submitted: 30 May 2019; Accepted for publication: 5 June 2019; Published online:

27 August 2019

Keywords: audit trail $\bullet$ data integrity $\bullet$ data transfers $\bullet$ EBF

The aim of this workshop was to increase the insight and understanding of regulatory expectations in relation to the new Medicines and Health products Regulatory Agency (MHRA) Guidance on GxP data integrity, issued in March 2018 [1]. The workshop also aimed to provide further insight and guidance on how to implement adequate levels of data integrity control dependent on the criticality of the data generated. The workshop was built with input and support from EBF expert members, who shared some of their recent MHRA inspection findings, and in collaboration with two MHRA inspectors. Finally, the workshop gave the participants, representing various parts of the bioanalytical community, an opportunity to discuss and define the current gap between the regulatory data integrity expectations and the availability of compliant software; highlighting the need for re-focus and commitment from the software developers to support users to attain the goal of having systems that meet the industry's data integrity requirements and the Regulator's expectations, based on relevant guidance and legislation.

\section{The Data Integrity Workshop}

The workshop was introduced by the organizing committee, on behalf of the EBF [2], to give a brief overview of the data integrity concepts and the overall intention and content of the workshop. This was followed by a presentation by one of the participating senior GCP and GLP inspectors from the MHRA, on their view on how to implement 
the data integrity guidance. The presentation highlighted the importance of understanding the terminology in the guidance as well as the basic concepts. The MHRA recognizes the technical, organizational and cultural challenges of implementing data integrity governance, and that there are no quick fixes. Implementation should be considered to be a multi-disciplinary activity and extensive changes may be required which will have resource and financial implications. It was accepted that change of this scale and potential complexity will require time to complete, but implementation of controls should be prioritized based on a data integrity risk assessment. The MHRA elaborated further on two main data integrity concepts:

- Data lifecycle; that includes data collection, processing, reporting, review and archiving;

- Data governance; that includes the process, the systems used and the ownership of these systems.

When defining these concepts, you should examine the role and access rights of all individuals, review all internal and external data transfers and examine whether there are any stages where data can be altered or deleted. Once these are defined and examined then the next steps are to decide what is critical data as all data are not equal. An example was provided; 'The acceptance criteria for calibration standards and quality controls (QCs)'. Should a user examine what software was used to produce this data? Who looks at the data? What are the potential risks in generating this data and can the data be manipulated or inadvertently changed? In addition, can a QC or calibration standard ever be reinjected and the data replaced?

The full slide set from the MHRA presentation can be found on the 11th EBF Open Symposium web page [3].

The workshop progressed to discuss and provide feedback on two main data integrity themes - Data Transfer/Control and Audit Trails. The organizing team introduced a number of questions on each theme to promote interactive discussions, which are summarized below:

Theme 1 - Data transfer/control:

- How can we transfer data and ensure no modifications and/or deletions occur in the process?

- What documentation can we store with our study file to ensure that data integrity is maintained at each data transfer step?

Theme 2 - Audit trail:

- How are audit trails used to ensure modifications and/or deletions of data are identified and captured in line with data integrity expectations?

- Why do we review the audit trail, what are the risks that we would like to mitigate?

- How do we document the outcome of the review?

- Should the audit trail review procedure be detailed in a standard operating procedure (SOP)?

In order to create an open and transparent atmosphere at the workshop and to set the scene for the round table discussions and panel, the above two themes were the primary focus in two case studies presented by two EBF member companies on recent MHRA inspection findings at their UK laboratory facilities.

In the first presentation, a summary of all the major findings was shared. This summary highlighted the challenge to the facility to ensure integrity and accuracy in the dataset when data acquired using for example the MassLyn ${ }^{\text {TM }}$ (MA, USA) software was transferred into Watson ${ }^{T M}$ LIMS (Thermo Fisher ${ }^{T M}$, MA, USA). In this case, the transfer process included the production of a text file that, if not protected from modifications, could be edited and then opened in the LIMS system with an amended dataset. In isolation, the quality assurance review of the data, in the format it resides in Watson LIMS (Thermo Fisher, MA, USA) and with flat file representation of the chromatograms printed to the NuGenesis ${ }^{\top M}$ (MA, USA) system, could not be considered to be representative of the raw data.

In a second presentation, similar data integrity deficiencies with laboratory analyzers between their LIMS system and MS software were presented as a finding from another recent MHRA inspection. This presentation also highlighted the MHRA's acceptance of their step-wise remediation plan, which included a substantial length of time to implement the changes to obtain the necessary data integrity controls. The slide sets from the two case study presentations can be found on the 11th EBF Open Symposium web page [3].

Following the introductory presentations and the two case studies, the workshop continued with interactive round table discussions where the approximately 100 workshop participants had the opportunity to discuss their specific data integrity issues in smaller groups. The main questions and concerns from these discussions as well as 
from the room in general were then addressed in a concluding panel discussion (comprising MHRA representatives, Laboratory Managers and Quality Assurance $[\mathrm{QA}]$ professionals), summarized under each theme below.

\section{Data transfer}

In the introduction given by the MHRA on implementation of data integrity controls, a process map exercise was suggested as a valuable tool to visualize and to define your 'data flow and process' universe. The process map(s) should help to define the systems, processes, equipment and roles impacted and to identify the key interfaces and communication lines. The process map(s) can also be used to evaluate the time, process and data criticality of each step. In the following panel discussion, the MHRA clarified that the process map(s) should be a tool for the business to visualize their business processes and that they should not be put in place 'just to please the regulators'. By understanding the use of the data in each step of the map, it is possible to identify the critical data as well as the controls and oversight measures required for adequate risk mitigation. The MHRA presentation also highlighted how data criticality should be determined by the intended use of the data, and that there is an expectation that risk management principles will be used to assess the risks and to identify the necessary mitigation steps. The Panel were asked if a 100\% QC check would ever be necessary for a manual/compromised data transfer step? In line with the previously conveyed risk management principles, the MHRA emphasized that the extent of any QC check would be dependent on the risks associated with the data generated. The importance of monitoring and documenting the outcome of any QC checking was also highlighted in order to verify if the applied QC process and its frequency was required and suitable.

During the round table discussions, the current lack of data integrity suitable software and interfaces was raised as a great concern. It was proposed that, as they become available, new software features should facilitate the data transfer process and possibly even standardize the integration functionality. The software vendor representatives who attended the workshop were not too optimistic about a full standardization; however, they did flag that there may be some 'low hanging fruit' that could be identified and/or implemented with improved software awareness and knowledge. One software feature suggested was to include a functionality that locked the path for the exported text files and then tracked the export event in the audit trail. Such a feature could allow for easy setup of a proper network area with no deletion permissions to significantly improve the data integrity. It is essential that as a community we push for enhanced data integrity control functionality.

As the discussions progressed the value of enhanced interaction and dialogue between the bioanalytical community and the software experts were also emphasized. Data integrity is now a primary focus for a bioanalytical laboratory when choosing new platforms/software and compliance must therefore also be considered by the software manufacturers when developing new software features. During the panel discussion, the MHRA reflected on this topic and pointed out the importance of knowing your software and having an awareness of what data integrity features are available; however, they recognized that not all instrument users were also experts on the software functionality. When purchasing new instrumentation data integrity features should be part of the evaluation. It was suggested that the vendor's knowledge of the system should be utilized to better understand the functionality available, for example, the terminology and interdependencies used within the security and permission modules and audit trails.

\section{Audit trail}

In the introduction by the MHRA they briefly touched upon audit trails and their expectation that everyone should know what audit trails were available on their system(s) as well as what they covered. Organizations should also know what audit trails are recording or not recording. A few questions were raised to gain the workshop participants' attention:

- Do you understand the terminology used in the audit trail?

- Who reviews audit trails and how regularly are they reviewed?

- Are these procedures documented in an SOP?

- Do QA understand what they are auditing?

- Can QA access and review data?

The MHRA highlighted the importance of understanding what 'normal' looks like in your audit trail in order to be able to identify any 'abnormal' activities within a reasonable timeframe and work effort. Finally, they shared 
some areas that are 'red flags' to inspectors. These were items such as full audit trails not being activated, standalone systems, manual data recording, manual data transfers and shared logins.

The MHRA data integrity expectation is, however, not a forensic approach, reviews will normally be targeted and driven by requirements covered in data integrity policies or SOPs. This was considered important because if the audit trail is only reviewed as part of the final QA, review problems will only be highlighted once the study has been completed.

The MHRA recognized the difficulties in preventing intentional fraud. To mitigate the risk of fraud, risk assessments should be made to identify weak points in a process and suitable measures put in place to make fraud more difficult to perpetrate and easier to detect. There is an acceptance that it is impossible to eliminate the risk of fraud and that residual risks will remain, but steps should be taken to minimize this risk.

\section{Conclusion \& future perspective}

The key take home messages from the workshop can be summarized as follows:

- Know your software system data and processes;

- Map your processes to identify the potential risks and weaknesses;

- Reduce the risk by implementing solutions that have been identified as a result of improved software awareness and knowledge;

- Open up the dialogue for enhanced interaction between system vendors, pharma/CROs and regulatory authorities to understand current, and define future, system data integrity capabilities.

The workshop was very well attended with approximately 100 participants, representing various roles (laboratory operators and management, quality, IT) from both Pharma and CRO companies. The broad audience, together with the open and energetic atmosphere at the workshop, highlights the great interest and engagement in this topic. The EBF e-environment team will therefore continue to drive the topic and arrange additional opportunities for dialogue on this topic, such as new interactive workshops. The EBF may investigate how we can extend the interaction to the broader instrument/software manufacturers to facilitate an improvement in the interface solutions. Also, potential synergistic activities with the EBF new technology and automation teams may be explored. From the workshop at the 11th Open Symposium, there is a clear desire to discuss how different companies have implemented their process mapping and risk analysis, and what has been learned during the planning, implementation and followup; EBF intends to create a platform for these experiences and learnings to be shared among the bioanalytical community and we would welcome contributions to this theme at the 12th EBF Open Symposium in Barcelona (20-22 November 2019).

\section{Acknowledgments}

The organizing committee would like to send a special thanks to all the presenters and panel members during the workshop - J Wakelin-Smith and A Gray (MHRA), S McDougall (ARCinova), M Lewis (GlaxoSmithKline) and D V Bedaf (Janssen R\&D). Thanks also to all the workshop participants for their contribution to an interactive and productive workshop.

\section{Disclaimer}

The views and conclusions presented in this paper are those of the European Bioanalysis Forum and do not necessarily reflect the representative affiliation or company's position on the subject.

\section{Financial \& competing interests disclosure}

The authors have no relevant affiliations or financial involvement with any organization or entity with a financial interest in or financial conflict with the subject matter or materials discussed in the manuscript. This includes employment, consultancies, honoraria, stock ownership or options, expert testimony, grants or patents received or pending, or royalties.

No writing assistance was utilized in the production of this manuscript. 


\section{References}

1. Medicines \& healthcare products regulatory agency (MHRA) - 'GXP' data integrity guidance and definitions (2018). https://assets.publishing.service.gov.uk/government/uploads/system/uploads/attachment_data/file/687246/MHR A_GxP_data_integrity_guide_March_edited_Final.pdf

2. European Bioanalysis Forum (EBF). http://www.e-b-f.eu

3. $11^{\text {th }}$ EBF Open Symposium slide decks: http://www.e-b-f.eu/bcn2018-slides/ 
